\title{
Microstructural analysis of Al6063/sic with calcium additives for hardness enhancement
}

\author{
Balaji Bakthavatchalam ${ }^{1, *}$, Khairul Habib ${ }^{1}$, Namdev Patil ${ }^{1}$, Omar A Hussein ${ }^{1}$ \\ ${ }^{1}$ Mechanical Engineering Department, Universiti Teknologi PETRONAS, 32610 Bandar Seri \\ Iskandar, Perak, Malaysia
}

\begin{abstract}
Microstructural Analysis plays an important role in enhancing the mechanical properties of metals and composites. Usually Aluminium Silicon Carbide (Al6063/SiC) alloys are mixed with strontium, sodium and antimony for high durability even though they are toxic and costly. As an alternative calcium is used as an alloying element to improve the mechanical property of Al6063/Sic alloy. In this paper Al6063 is chosen as the matrix material while Sic is used as a reinforcement where calcium powder is added to modify the silicon phase of the composite. Finally, concentration of Silicon carbide is varied from 0 to $150 \mathrm{mg}$ to produce four specimens of Al6063 alloy and it is subjected to microstructure analysis which showed the reduction of grain size and therefore improvement in the hardness from 52.9 $\mathrm{HV}$ to $58.4 \mathrm{HV}$ and decrease in the wear loss from 3.97 to 3.27 percentage.
\end{abstract}

\section{Introduction}

Every manufacturing industry have a consistent strain to fabricate a good quality product with less cost. In this way they required another innovation in expanding the generation with a high caliber which can be fulfilled by composite materials. A composite material is a material made out of at least two distinct phases (dispersed phase and matrix phase) and has a comprehensive property that is fundamentally not the same as any segment. The matrix phase is the principle phase with persistent highlights. The framework is generally more pliable and has a lower hard phase. It keeps up the dispersed phase and offers the heap with it. The second phase is inserted in the framework in a broken form. This second phase is known as the dispersed phase which is typically more grounded than the matrix, so it is now and then called the fortification or reinforcing phase [1]. A large number of normal materials (metal amalgams, doped Ceramics and Polymers blended with added substances) likewise have a little quantity of scattered phases in their structures, anyway they are not considered as composite materials since their properties are like those of their base constituents.

Shakoori et al. analyzed the yield strength of bimodal Al6063 alloy by a linear superposition hardening from grating friction, high-angle boundaries, low-angle boundaries and particles of the second phase. The predicted yield strengths were in fairly good agreement with experimental data [2]. Idusuyi et al. studied the effects of chromium on the electrochemical behavior of Al6063 which revealed that corrosion performance of Al6063

* Corresponding author: balaji_17004028@utp.edu.my 
was optimal at 3 weight \% chromium implying a greater resistance to corrosion [3]. Prashantha et al. concluded that the Material Removal Rate (MRR) decreases with increasing percentage of volume fractions of silicon carbide particles in Metal Matrix composites and the average MRR for Al606 is 9.15, 9.13 and $9 \mathrm{~mm} 3 / \mathrm{min}$ with 3\%, 6\% and $9 \%$ Sic respectively [4]. Hafeez et al. depicted the auxiliary advancement related with grain refinement and predominant mechanical properties by the expansion of nano-size $\mathrm{Al} 2 \mathrm{O} 3$ and Y2O3 particulates into solid Al6063 combination grid. Results demonstrate that the $0.2 \%$ yield quality $(0.2 \%$ YS), Ultimate Tensile Strength (UTS), work of fracture (WOF) were enhanced over the unreinforced solid A16063 amalgam and failure strain was diminished in fortified nano-composites [5]. Based on the above researches, it is clear that the strength of Al6063 alloy can be increased by using additives. The focus of this research is to find the effect of calcium and silicon carbide in terms of microstructure, hardness and abrasion loss on the fabricated Al6063 Alloy.

\section{Experimental materials and test procedure}

Many researchers have been working on modification of aluminium alloys, majority have been concerned with explaining how it is possible to change eutectic silicon from coarse plates to fine fibers. There are almost certainly other elements, which are capable of changing the form of eutectic silicon in aluminium casting alloys. But for the moment, only sodium, strontium and antimony find only significant industrial use. Modification in hypoeutectic silicon alloy tend to reduce the surface tension within the eutectic phase which consists of a rather lamellae structure of eutectic aluminium and eutectic silicon. The reduction in surface tension increases the contact angle between aluminium and silicon allowing the aluminium to periodically envelop and arrest the growth of silicon crystal. The amount of each element required depends somewhat on the alloy composition where higher silicon content requires more modifying agent.

Strontium, Sodium and Antimony are commonly used for modification in Al alloys. Sodium is a reactive material. It can react when it is exposed to air and can burn violently during addition to molten aluminium. Antimony is a toxic material, so it has some risk during handling. Strontium is a costlier material when compared to Calcium. Calcium is used as wetting promoters in the synthesis of aluminium metal matrix composites. As an alloying element calcium can also impart super plasticity. Calcium refines the grains of the aluminium alloy. Calcium modifiers are not commercially used. So in this project the calcium is used as a modifier in $\mathrm{Al}$ and $\mathrm{SiC}$ composite to find the properties change.

\subsection{Composite preparation}

Al6063 is chosen as the matrix material and $\mathrm{SiC}$ is chosen as reinforcement for composite making. First, a crucible made of clay and graphite was placed inside the furnace and preheated to a temperature about $200^{\circ} \mathrm{C}$. Preheating was done at $200^{\circ} \mathrm{c}$ to drive off unwanted moisture. About half $\mathrm{kg}$ of A16063 alloy was placed inside the crucible. When the alloy melting point reaches around $760^{\circ} \mathrm{C}$, the furnace was turned $\mathrm{OFF}$.

An impeller, which was driven by an electric motor through a shaft, was centrally placed into the melt. The impeller was rotated to form the vortex in the melt. Now the furnace is again turned $\mathrm{ON}$. When the temperature reaches around $740^{\circ} \mathrm{C}$ predetermined quantity of preheated silicon carbide particles of $250 \mu \mathrm{m}$ were gradually added to the melt through an inclined pipe. After addition of the particles the melt was stirred for 10 minutes.

After 10 minutes the stirring was stopped and the melt is superheated to $760^{\circ} \mathrm{C}$. The melt was held at this temperature for about 5 minutes. Then it was poured into the mould for making specimens. The crucible containing the melt was again placed inside the furnace and 
heated to a temperature of about $730^{\circ} \mathrm{C} .0 .04$ wt. $\%$ of calcium metal powder was added for the purpose of modification. After the addition of calcium, the melt was heated for 10 minutes. The liquid metal was then poured into the mould. By using the above process, composites containing different weight percent $(5,10$, and 15) of silicon carbide particles were prepared for modified and unmodified conditions.

\subsection{Testing methods}

The casted specimens are subjected to various tests for analysis. They are follows

- The microstructures of specimens are studied through metallographic studies.

- The hardness properties of the specimens are studied through conducting harness test in hardness testing machine.

- Wear test is conducted using wear resistance tester machine.

\subsubsection{Metallographic study}

Samples of small squares having the sides of $15 \mathrm{~mm}$ were cut from the rectangular specimens and small cylinders having a height of $5 \mathrm{~mm}$ were cut from cylindrical specimens. First for rough polishing, specimen was ground on various grades $(80,100,220,400)$ of emery paper. The specimens were thoroughly washed in running water before going from one grade paper to another grade. Grinding was carried out in each grades of paper until the scratches made by previous paper disappeared. For this, the specimen was of the paper. After rough polishing, fine polishing was done with Sclvyt cloth. The medium used for polishing was kerosene. The polished specimen was etched using the hydro fluoric acid and washed thoroughly in running water. Using a hot air drier, the specimens were dried. After drying, photographs of microstructure were taken.

\subsubsection{Hardness test}

Hardness specimens of $15 \mathrm{mmX} 15 \mathrm{~mm}$ were taken from rectangular specimens and $5 \mathrm{~mm}$ height specimens were taken from cylindrical specimens. The top surface of the specimens was polished using emery paper. The specimen was then placed on the anvil of the tester and a steel ball indenter of $5 \mathrm{~mm}$ diameter was used to apply load. A load of $250 \mathrm{KN}$ was applied for the duration of 30 seconds and the diameter of the indentation was measured with a low power microscope after removal of the load. The micro Vickers hardness tester can be expressed as the load divided by the surface area of the indentation.

\subsubsection{Wear resistance test}

A sheet of specified abrasive paper $(60 \mathrm{Grid})$ is fixed to the drum. The cylinder sample of fixed dimension is inserted into the sample holder and the drum holder is rotated with sample traveling over the surface of the abrasive paper which makes the drum to rotate for 84 times i.e. 40 meters long. After completion of the test, the sample is removed and the volume loss is determined by weight calculation for the four test specimens and the result are expressed in mean value.

\section{Results and discussion}

\subsection{Microstructural analysis}


Microstructure of a material gives a good indication of mechanical properties of that material. Any change in properties can attribute to a corresponding change in the microstructure of that material. In the microstructure of cast modified, unmodified alloys and composites Small Island like structures represents aluminium dendrite while small green spots represent the silicon carbide distribution. Comparing the microstructures of modified and unmodified alloys, it can be seen that the aluminium dendrites has been fined as a result of calcium modification. This fining of dendrites aluminium is achieved by promoting the growth of aluminium dendrites, which is achieved by calcium modification. Increase in SiC particles composition can be seen from the microstructure of composites having higher particulate content. It is visible from the microstructure of composites that the effect of calcium is much more pronounced in composites with higher $\mathrm{SiC}$ particles than in composites having lesser $\mathrm{SiC}$ particle composition. When compared to unmodified cast alloys, the grains of modified cast alloys form a uniform structure. Images of the microstructure of specimens are given below

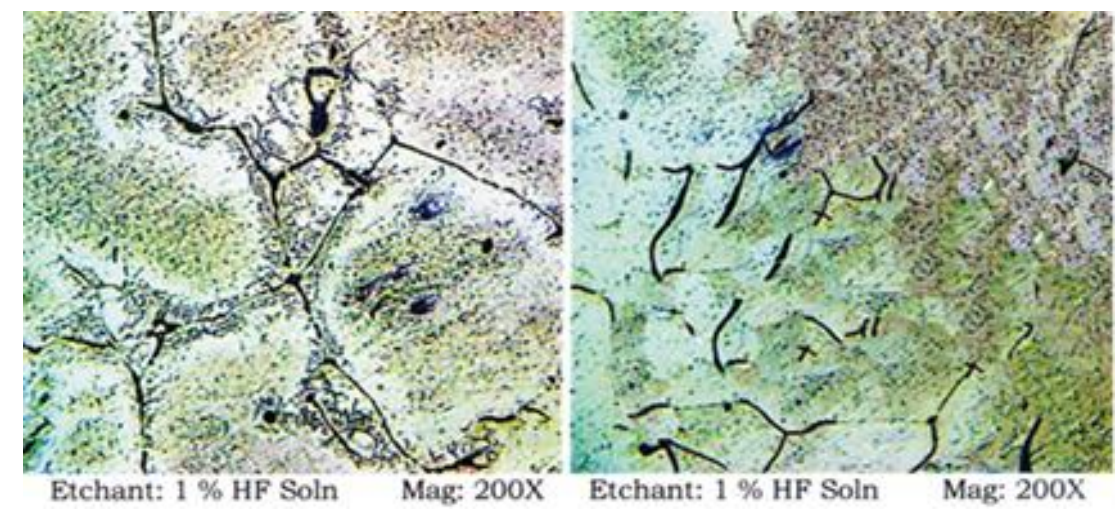

Fig. 1. Microstructure Sample of $[\mathrm{A} 16063+\mathrm{TiO} 2+\mathrm{Ca}]$

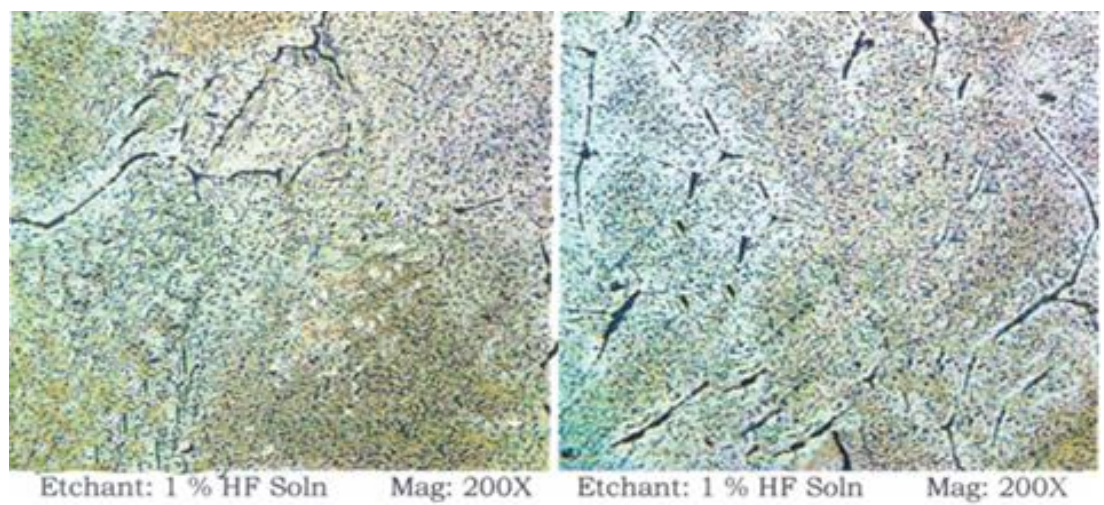

Fig. 2. Microstructure Sample of [ $\mathrm{A} 16063+\mathrm{TiO} 2+\mathrm{Ca}+\mathrm{SiC}(50 \mathrm{gm})]$ 


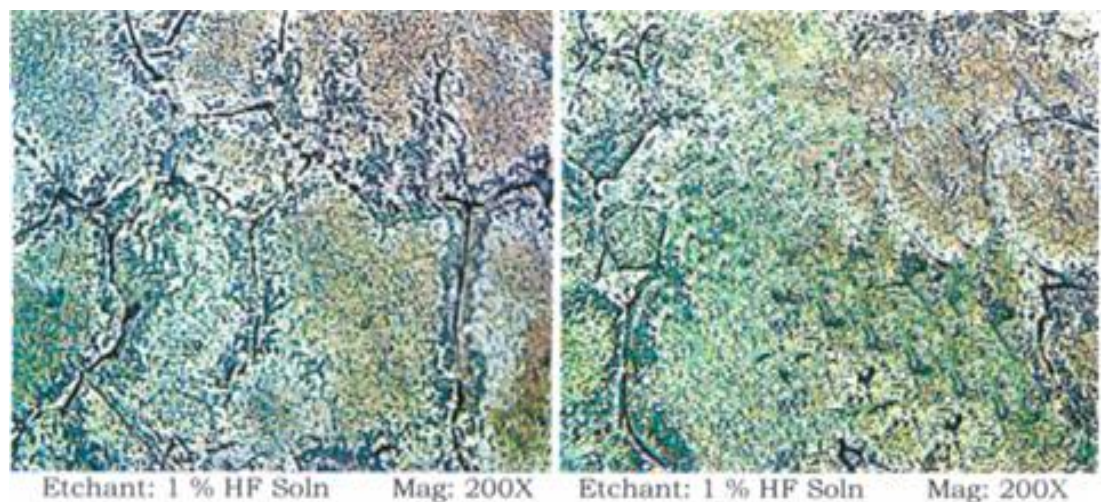

Fig. 3. Microstructure Sample of $[\mathrm{Al} 6063+\mathrm{TiO} 2+\mathrm{Ca}+\mathrm{SiC}(100 \mathrm{gm})]$

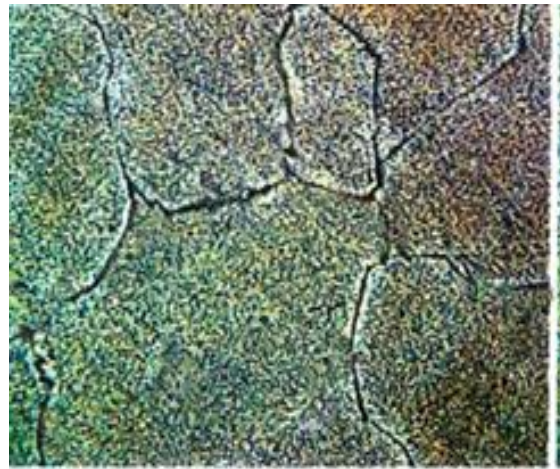

Etchant: $1 \%$ HF Soln
Mag: $200 \mathrm{X}$

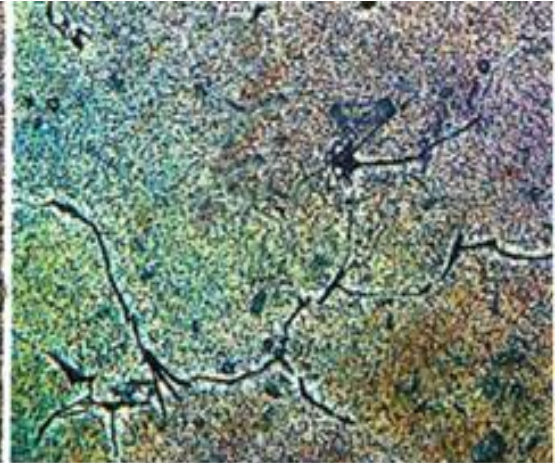

Etchant: $1 \% \mathrm{HF}$ Soln

Mag: $200 \mathrm{X}$

Fig. 4. Microstructure Sample of [A16063+TiO2+Ca+SiC (150gm)]

Strontium modification coarsened the aluminium dendrites. But Calcium modification fines the aluminium dendrites. Strontium modified composites have higher porosity than Calcium modified composites. Comparatively Strontium metal has higher cost than Calcium metal. Moreover, Strontium metal is a rare material.

\subsection{Hardness tests}

The table below compares the hardness of modified and unmodified cast alloys

Table 1. Hardness test result

\begin{tabular}{|c|c|c|c|}
\hline SAMPLES & $\begin{array}{c}\mathbf{2 0 0} \text { KN } \\
\text { (HV) }\end{array}$ & $\begin{array}{c}\mathbf{2 2 5} \text { KN } \\
\text { (HV) }\end{array}$ & $\begin{array}{c}\mathbf{2 5 0} \text { KN } \\
\text { (HV) }\end{array}$ \\
\hline AL6063+TiO2+Ca-B & 53.5 & 53.2 & 52.9 \\
\hline AL6063+TiO2+Ca+SiC(50g) & 55.5 & 55.3 & 55.1 \\
\hline AL6063+TiO2+Ca+SiC(100g) & 56.8 & 56.8 & 56.5 \\
\hline AL6063+TiO2+Ca+SiC(150g) & 58.4 & 58.2 & 58.2 \\
\hline
\end{tabular}




\subsection{Wear test}

Abrasive wear is carried out using wear tester machine. Results are shown as follows

Table 2. Wear test result

\begin{tabular}{|c|c|c|c|c|}
\hline SAMPLES & $\begin{array}{c}\text { INITIAL } \\
\text { WEIGHT(g) }\end{array}$ & $\begin{array}{c}\text { FINAL } \\
\text { WEIGHT(g) }\end{array}$ & $\begin{array}{c}\text { ABRASION } \\
\text { LOSS (g) }\end{array}$ & $\begin{array}{c}\text { Wear } \\
\text { Resistance( \%) }\end{array}$ \\
\hline $\begin{array}{c}\mathrm{AL} 6063+\mathrm{TiO}_{2}+\mathrm{Ca}- \\
\mathrm{B}\end{array}$ & 9.1251 & 8.7626 & 0.3625 & 3.97 \\
\hline $\begin{array}{c}\mathrm{AL6063}+\mathrm{TiO}_{2}+\mathrm{Ca}+ \\
\mathrm{SiC}(50 \mathrm{~g})\end{array}$ & 10.1744 & 9.8054 & 0.3690 & 3.63 \\
\hline $\begin{array}{c}\mathrm{AL} 6063+\mathrm{TiO}_{2}+\mathrm{Ca}+ \\
\mathrm{SiC}(100 \mathrm{~g})\end{array}$ & 9.1517 & 8.7917 & 0.3600 & 3.43 \\
\hline $\begin{array}{c}\mathrm{AL} 6063+\mathrm{TiO}_{2}+\mathrm{Ca}+ \\
\mathrm{SiC}(150 \mathrm{~g})\end{array}$ & 8.9516 & 8.5869 & 0.3647 & 3.27 \\
\hline
\end{tabular}

\section{Conclusion}

The following conclusions are drawn from this project

- Al6063/SiC composites with varying amount of $\mathrm{SiC}$ particle composition (5\%, 10\%, and $15 \%$ ) under modified conditions have been successfully made by stir casting.

- Microstructure comparison of modified alloy was made. From the results, it is observed that calcium addition has resulted in the reduction of grain size and therefore improved hardness and strength.

- It can be noted that the increase in the content of $\mathrm{SiC}$ particles in the composite increases the hardness of the modified composite from $53.5 \mathrm{HV}$ to $58.4 \mathrm{HV}$ and reduces the wear of the modified composite from 3.97 to $3.27 \%$.

\section{References}

1. Suganthi Rekha, V.K.Bupesh Raja, Journal of Material Science and Engineering 197, 6 (2017)

2. M. Shakoori Oskooie, H. Asgharzadeh, H.S. Kim, Journal of Alloys and compounds 229, 15 (2018)

3. N. Idusuyi, O. Ajide, O. Oluwole, O.A. Arotiba, Proceedia Manufacturing 7, 413(2016)

4. S. Prashantha, B. Veeresha, S.M. Shashidhara, Mallikarjun, Materials Today Proceedings, 4, 10779(2017)

5. Hafez Ahamed, V. Senthilkumar, H.S. Kim, Journal of Material and Design 37, 182 (2012)

6. Zhi Yong Cai, Chun Zhang, Ri Chu Wang, Chao Qun Peng, Ke Qui, Transactions of Nanoferrous Metals Society of China 26, 2355(2016) 